\title{
L-Performance with an Application to Hedge Funds
}

Serge Darolles*

Christian Gourieroux ${ }^{\dagger}$

Joann Jasiak $\ddagger$

This version: June 26, 2008

*SGAM AI and CREST, e-mail: serge.darolles@sgam.com.

${ }^{\dagger}$ CREST, CEPREMAP and University of Toronto, e-mail: gouriero@ensae.fr.

${ }^{\ddagger}$ York University, Canada, e-mail: jasiakj@yorku.ca

This study benefited from financial support of the Natural Sciences and Engineering Council of Canada (NSERC), the Chair AXA "Large Risks in Insurance" and the Chair GROUPAMA "Behavioral Finance". 


\begin{abstract}
L-Performance with an Application to Hedge Funds

This paper introduces a new fund performance measure, called the Lperformance. It is proposed as an alternative to the Sharpe performance measure that is commonly used for fund performance valuation despite its inability to account for the skewness and thick tails of fund return distributions. The L-performance improves upon the Sharpe measure in this respect. Technically, the L-performance is based on sample statistics, called L-moments, which are conceptually close to the conventional power moments, but provide more detailed information about the extremes. For this reason, the L-moments are used for prediction and assessment of extreme events, such as floods and earthquakes. In this paper, the new L-performance measure is calculated for a variety of hedge funds and is used to derive a fund ranking.
\end{abstract}

Keywords: Hedge Fund, Sharpe Performance, L-moment, Distortion Risk Measure, Ranking, Bias Ratio, Manipulation.

JEL number: . 


\section{Introduction}

The so-called Sharpe performance is a commonly accepted measure of fund performance. It is defined as a ratio of the expected excess return and volatility, and used for segmentation and fund ranking [see e.g. Sharpe (1966), Lo (2002), Darolles, Gourieroux (2008)]. Its main shortcoming is that it relies on only two statistics, i.e. the sample mean and volatility, which do not always provide a sufficiently precise characterization of return distributions, especially when these distributions feature skewness and thick tails. In particular, the sample mean may not provide a sufficiently robust approximation of the location parameter, and the standard error may not properly account for the size of tails. This is of special importance for the (unconditional) distributions of hedge fund returns, whose departures from normality are related to some liquidity and management characteristics [see e.g. Getmansky, Lo, Makarov (2003)].

The aim of this paper is to introduce a battery of alternative performance measures which are robust to outliers and can accommodate the thick tailed distributions. The new measures are based on the notion of trimmed L-moments, used in the analysis of catastrophic events such as extreme floods or low flows [see e.g. Hosking, Wallis (1987), Wang (1997), Bayazit, Onoz (2002), Moisello (2007)], rainfall extremes [see e.g. Guttman et al. (1993), Lee, Maeng (2003)], extreme wind speed [see e.g. Whalen et al. (2004)], or extreme traffic volumes in computer networks [see e.g. Hosking (2007)]. It seems that there exists only two published applications of L-Moments to financial returns [Karvanen (2006), Gourieroux, Jasiak (2008)].

In Section 2, we recall and extend the definitions of trimmed L-moments of orders 1 and 2, and discuss their interpretations in terms of the quantile and concentration functions. The L-performances are defined in Section 3 as ratios of trimmed L-moments of order 1 and 2. We explain how the L-performances are estimated and derive the asymptotic distribution of the L-performance estimator. The performance measures are used in Section 4 to rank a set of hedge funds. Section 5 concludes. Proofs are gathered in Appendices. 


\section{Trimmed L-Moments}

The notion of a trimmed L-moment has been introduced in Elamir, Seheult (2003) as an extension of the L-moments studied in Hosking (1990). Let us consider a continuous random variable $X$ with positive probability density function (pdf) $f$, cumulative distribution function (cdf) $F$ and quantile function $Q=F^{-1}$. The trimmed L-moments are defined from a so-called "conceptual random sample" of size $2 n+1$, drawn independently from distribution $\mathrm{F}$. The variables in the conceptual sample $\tilde{X}_{1}, \ldots, \tilde{X}_{2 n+1}$ are ranked in an ascending order, providing the order statistics $\tilde{X}_{1: 2 n+1}<\tilde{X}_{2: 2 n+1}<\ldots<\tilde{X}_{2 n+1: 2 n+1}$. In particular, the sample minimum (resp. maximum) is $\tilde{X}_{1: 2 n+1}$ (resp. $\tilde{X}_{2 n+1: 2 n+1}$ ).

\subsection{Trimmed L-moments of order 1}

The $n$-trimmed L-moment of order 1 is defined by:

$$
\lambda_{1, n}=E\left(\tilde{X}_{n+1: 2 n+1}\right), n \geq 0 .
$$

It is equal to the expectation of the median of conceptual sample. The trimmed L-moment of order 1 of $X$ has the following expressions involving the cdf and the quantile function, respectively [Elamir, Seheult (2003), Hosking (2007)]:

$$
\begin{aligned}
\lambda_{1, n} & =\frac{(2 n+1) !}{(n !)^{2}} \int x F(x)^{n}[1-F(x)]^{n} f(x) d x \\
& =\frac{(2 n+1) !}{(n !)^{2}} \int_{0}^{1} Q(u) u^{n}(1-u)^{n} d u .
\end{aligned}
$$

The polynomials

$$
P_{1, n}(u)=\frac{(2 n+1) !}{(n !)^{2}} u^{n}(1-u)^{n},
$$

are nonnegative with unit mass. The polynomials $P_{1, n}$ define probability distributions, called the distortion measures [Wang (2000a), (2000b), Gourieroux, Liu (2007)]. For a fixed $n$, moment $\lambda_{1, n}$ is a weighted average over the quantile function with higher weights assigned to arguments $u$ close to 0.5 [see Greenwood et al. (1979) for this interpretation]. For $n=0$, the mean is obtained: $\lambda_{1,0}=\int_{0}^{1} Q(u) d u=E X$ and, when $n$ tends to infinity, the median is obtained: $\lambda_{1, \infty}=\lim _{n \rightarrow \infty} \lambda_{1, n}=Q(0.5)$. When $n$ increases, the trimmed 
L-moments of order 1 bridge the mean and the median. The existence of trimmed Lmoments requires less restrictions than the existence of conventional moments. Indeed, moment $\lambda_{1, n}$ exists if $E\left(|X|^{1 /(n+1)}\right)<\infty$ [see Hosking (2007), Theorem 1]. Thus, the trimmed L-moments for $n \geq 1$ can be defined even when the expectation of returns does not exist, and they are less sensitive to outliers when $n$ increases.

The patterns of polynomials $P_{1, n}$ are displayed in Figure 1.

[Insert Figure 1: Weighting Polynomials $P_{1, n}$ ]

The distortion measure associated with polynomial $P_{1, n}$ is symmetric, centered at 0.5 , and becomes more peaked when $n$ increases. In contrast, it tends to a uniform distribution (resp. point mass at 0.5 ), when $n$ tends to 0 (resp. infinity).

\subsection{Trimmed L-moments of order 2}

The $(r, n)$ trimmed L-moment of order 2 is defined by ${ }^{1}$ :

$$
\lambda_{2, r, n}=E\left(\tilde{X}_{2 n-r+1: 2 n+1}-\tilde{X}_{r+1: 2 n+1}\right), 0 \leq r \leq n-1 .
$$

It measures the expected range of a conceptual sample after deleting the $r$ smallest and $r$ largest conceptual observations. These measures increase with $r$. This L-moment of order 2 exists when $E\left[|X|^{1 /(r+1)}\right]<\infty$ [see Hosking (1990)]. Thus, the measures are less sensitive to outliers when $r$ increases. Definition (2.4) extends the concept introduced in Elamir, Seheult (2003), which corresponds to the special case $r=n-1$, and $\lambda_{2, n-1, n}=$ $E\left(\tilde{X}_{n+2: 2 n+1}-\tilde{X}_{n: 2 n+1}\right)$. For this particular value of $r$, the conceptual range is determined by variables with ranks $n$ and $n+2$. In financial applications, it seems preferable to choose a value of $r$ much smaller than $n-1$ in order to better capture extreme risks.

The analytical expression of the trimmed L-moment of order 2 is:

$$
\lambda_{2, r, n}=\frac{(2 n+1) !}{r !(2 n-r) !} \int x\left\{F(x)^{2 n-r}[1-F(x)]^{r}-F(x)^{r}(1-F(x)]^{2 n-r}\right\} f(x) d x
$$

\footnotetext{
${ }^{1}$ The definition above assumes an odd size of the virtual sample. This definition does not include the basic L-moment of order 2 initially introduced by Hosking (1990). This L-moment is:

$\lambda_{2}=E\left(\tilde{X}_{2: 2}-\tilde{X}_{1: 2}\right)=\int_{0}^{1} Q(u)(2 u-1) d u$.

If we denote $G(u)=\int_{0}^{1} Q(x) d x$ the cumulated quantile function, that is the Gini (or concentration) curve [Gini (1912)], we get by integrating by part: $\lambda_{2}=\int_{0}^{1}(2 u-1) d G(u)=G(1)-2 \int_{0}^{1} G(u) d u$.
} 


$$
=\frac{(2 n+1) !}{r !(2 n-r) !} \int_{0}^{1} Q(u)\left[u^{2 n-r}(1-u)^{r}-u^{r}(1-u)^{2 n-r}\right] d u .
$$

The polynomials:

$$
P_{2, r, n}(u)=\frac{(2 n+1) !}{r !(2 n-r) !}\left[u^{2 n-r}(1-u)^{r}-u^{r}(1-u)^{2 n-r}\right]
$$

can be of any sign and have mass zero. Thus the associated distortion measures are not positive. The patterns of polynomials $P_{2, r, n}$ vary with $n$ and $r$, as shown in Figures 2 and 3 .

[Insert Figure 2: Polynomials $P_{2, r, n}$ for $r=1$ and varying $n$ ]

[Insert Figure 3: Polynomials $P_{2, r, n}$ for $n=5$ and varying $r$ ]

The polynomials introduce shrinkages in the neighborhoods of values $u=0,1$ and 0.5 . These shrinkages are controlled by the selected values of parameters $r$ and $n$. It follows that, when $n \rightarrow \infty, r \rightarrow \infty$, with $r /(2 n+1) \rightarrow \alpha$, the distortion measure tends to $-\epsilon_{1-\alpha}+\epsilon_{\alpha}$, where $\epsilon_{1-\alpha}, \epsilon_{\alpha}$ denote the point mass at $1-\alpha$ and $\alpha$, respectively, and the trimmed L-moment of order 2 tends to $^{2} Q(1-\alpha)-Q(\alpha)$. This is the difference between an upper- and a lower tail $\alpha$-quantiles. For example, they can be calculated for a small $\alpha$, such as $\alpha=1 \%, 5 \%$ or $10 \%$.

The trimmed L-moments at order 2 are dispersion measures. They measure a slope of the quantile function in the limiting case $n=\infty$ (resp. the expected slope of the empirical virtual quantile function, in general). By decreasing the ratio $r /(2 n+1)$, we reveal the differences between the right- and left-tail extremes. The trimmed L-moments are larger if the slope is larger, or equivalently if the cumulative distribution function is less flat [about the median since $\alpha+(1-\alpha)=1]$.

The L-moment at order 2 has an integral representation equivalent to (2.5) and based on the cumulated quantile function $G(u)=\int_{0}^{u} Q(x) d x$. Function $G$ is the concentration (or Lorentz) curve used in concentration analysis and in stochastic dominance of order 2. By integrating by part, we get:

$$
\lambda_{2, r, n}=\int_{0}^{1} P_{2, r, n}(u) d G(u)
$$

\footnotetext{
${ }^{2}$ When $r=n-1$ as in Elamir, Sehenlt (2003) or Hosking (2007), the ratio $r /(2 n+1)$ tends to $1 / 2$ when $n$ tends to infinity, and the trimmed L-moment of order 2 tends to $Q(1 / 2)-Q(1 / 2)=0$.
} 


$$
\begin{aligned}
& \left.=P_{2, r, n}(u) G(u)\right]_{0}^{1}+\int_{0}^{1} G(u)\left[-\frac{d P_{2, r, n}}{d u}(u)\right] d u \\
& =\int_{0}^{1} G(u)\left[-\frac{d P_{2, r, n}}{d u}(u)\right] d u .
\end{aligned}
$$

In Figure 2, we see that the L-moment assigns positive weights to the extremes (i.e. the values of $u$ close to 0 and 1), and thus focuses on extreme risks. Equivalently, the L-moment of order 2 can also be written in terms of covariance, since $\lambda_{2, r, n}=\operatorname{cov}\left(X, P_{2, r, n}[F(X)]\right)$. It measures the link between the return and its (historical) rank [Serfling, Xiao (2006)].

\section{L-Performance}

Let us now consider a sequence $x_{1}, \ldots, x_{T}$, say, of portfolio excess returns. In this paper we consider the historical, i.e. the unconditional, measure of performance, in line with the hedge fund literature and the publicly available hedge fund ratings. For ease of exposition we assume ${ }^{3}$ that returns are independent and identically distributed (iid) with a distribution characterized by $\operatorname{cdf} F$ and quantile function $Q=F^{-1}$. The associated observed order statistics are: $x_{1: T} \leq \ldots \leq x_{T: T}$. The observed order statistics have to be distinguished from the conceptual order statistics $\tilde{X}_{1: 2 n+1}<\ldots<\tilde{X}_{2 n+1 ; 2 n+1}$ introduced in Section 2. The latter ones are artificial, and are used to define the trimmed L-moment of interest. A priori, there is no relation between the shrinkage parameters $r, n$, which define the theoretical moment of interest, and the number of observations $T$.

\subsection{Definition}

By analogy to the Sharpe performance ratio, which is the ratio of the expected net return and volatility [Sharpe (1966)], the L-performance is defined as the ratio of a trimmed L-moment of order 1 and a trimmed L-moment of order 2 :

$L_{r, n}=\lambda_{1, n} / \lambda_{2, r, n}$

\footnotetext{
${ }^{3}$ For the standard Sharpe performance measures, two extensions to non-iid returns are possible:

i) First, one can still consider the historical performance measures, and in addition derive their asymptotic distributions in a non-iid framework [see e.g. Lo (2002) for this approach applied to Sharpe performance].

ii) Second, one can consider the conditional performance measures instead of unconditional ones [see e.e. Darolles, Gourieroux (2008) for conditional Sharpe performances and Gourieroux, Jasiak (2008) for the definition of conditional L-Moments].

These extensions are clearly out of the scope of the present paper.
} 


$$
=\frac{r !(2 n-r) !}{(n !)^{2}} \int_{0}^{1} Q(u) u^{n}(1-u)^{n} d u\left[\int_{0}^{1} Q(u)\left[u^{2 n-r}(1-u)^{r}-u^{r}(1-u)^{2 n-r}\right] d u\right]^{-1} .
$$

This leads to a battery of L-performances that depend on the selected shrinkage parameters $r$ and $n$. For example, we have:

$$
\begin{aligned}
L_{0,1} & =2 \int_{0}^{1} Q(u) u(1-u) d u\left[\int_{0}^{1} Q(u)(2 u-1) d u\right]^{-1} \\
L_{1,2} & =3 / 2 \int_{0}^{1} Q(u) u^{2}(1-u)^{2} d u\left[\int_{0}^{1} Q(u)\left[u^{3}(1-u)-u(1-u)^{3}\right] d u\right]^{-1} \\
& =3 / 2 \int_{0}^{1} Q(u) u^{2}(1-u)^{2} d u\left[\int_{0}^{1} Q(u) u(1-u)(2 u-1) d u\right]^{-1}
\end{aligned}
$$

When $n \rightarrow \infty, r \rightarrow \infty$, and $r /(2 n+1) \rightarrow 5 \%$, say, we have:

$$
\lim _{r, n \rightarrow \infty} L_{r, n}=\frac{Q(50 \%)}{Q(95 \%)-Q(5 \%)}=\frac{\operatorname{VaR}(50 \%)}{\operatorname{VaR}(95 \%)-\operatorname{VaR}(5 \%)},
$$

where VaR denotes the Value-at-Risk computed by historical simulation as suggested in the standard approach by Basle Committee. In fact, the L-performance measures extend the (inverse) Gini concentration index [Gini (1912)]. Indeed $\lambda_{2} / \lambda_{1}=1-2 \int_{0}^{1} G(u) d u / G(1)$ is exactly the Gini index formula.

When the returns are such that $x_{t}=m+\sigma u_{t}$, where the error terms have a symmetric distribution and $m, \sigma$ are the location and scale parameters, respectively, we have: $Q(u)=$ $m+\sigma G(u)$, where $G$ is the quantile function of the standardized error term. By the symmetry condition, we have: $G(-u)=G(1-u)$. It follows that:

$$
L_{r, n}=\frac{r !(2 n-r) !}{(n !)^{2}} \frac{m}{\sigma}\left[\int_{0}^{1} G(u)\left[u^{2 n-r}(1-u)^{r}-u^{r}(1-u)^{2 n-r}\right] d u\right]^{-1}
$$

In this framework, the L-performances are proportional to the ratio $m / \sigma$, up to a scale factor depending on $r, n$, and on the error term distribution. In particular, for Gaussian errors, the L-performance is equivalent to the standard Sharpe performance measure, up to a multiplicative scalar function of the shrinkage parameters only. 


\subsection{Estimation of L-performance}

The L-performances are easily estimated from their sample counterparts. The estimator is defined by (see Appendix 1):

$$
\hat{L}_{r, n, T}=\frac{\sum_{t=1}^{T} x_{t: T} P_{1, n}(t / T)}{\sum_{t=1}^{T} x_{t: T} P_{2, r, n}(t / T)} .
$$

This is a ratio of two linear combinations of order statistics ${ }^{4}$. These estimators are consistent, asymptotically normal under standard regularity conditions (see Appendix 1). For instance, for independent and identically distributed ${ }^{5}$ excess returns, we have:

$$
\sqrt{T}\left(\hat{L}_{r, n, T}-L_{r, n}\right) \stackrel{d}{\rightarrow} N\left(0, \eta_{r, n}^{2}\right),
$$

where the asymptotic variance is given by:

$\eta_{r, n}^{2}=\lambda_{2, r, n}^{-2} \int_{0}^{1} \int_{0}^{1} \frac{\min \left(u_{1}, u_{2}\right)-u_{1} u_{2}}{f\left[Q\left(u_{1}\right)\right] f\left[Q\left(u_{2}\right)\right]}\left[P_{1, n}\left(u_{1}\right)-L_{r, n} P_{2, r, n}\left(u_{1}\right)\right]\left[P_{1, n}\left(u_{2}\right)-L_{r, n} P_{2, r, n}\left(u_{2}\right)\right] d u_{1} d u_{2}$.

\subsection{Estimation of the asymptotic variance of $\hat{L}_{r, n, T}$}

The asymptotic variance can be alternatively written as:

$$
\begin{aligned}
\eta_{r, n}^{2}= & \lambda_{2, r, n}^{-2} \iint \min \left(F\left(x_{1}\right), F\left(x_{2}\right)\right)-F\left(x_{1}\right) F\left(x_{2}\right)\left[P_{1, n}\left(F\left(x_{1}\right)\right)-L_{r, n} P_{2, r, n}\left(F\left(x_{1}\right)\right)\right] \\
& {\left[P_{1, n}\left(F\left(x_{2}\right)\right)-L_{r, n} P_{2, r, n}\left(F\left(x_{2}\right)\right)\right] d x_{1} d x_{2} }
\end{aligned}
$$

by applying the change of variable $x=Q(u)$, and given that $1 / f[Q(u)]$ is the derivative of the quantile function $Q$.

The sample counterpart of the expression above provides a consistent estimator of the asymptotic variance [see e.g. Jones, Zitikis (2003), (2005)]:

\footnotetext{
${ }^{4} \mathrm{~A}$ linear combination of order statistics is called a L-statistic in the statistical literature. This justifies the terminology "L-moment" and "L-performance".

${ }^{5}$ The asymptotic behavior can also be derived for stationary, serially dependent excess returns [see e.g. Gourieroux, Liu (2007)].
} 


$$
\begin{aligned}
\hat{\eta}_{r, n, T}^{2}= & \hat{\lambda}_{2, r, n, T}^{-2} \sum_{i=1}^{T-1} \sum_{j=1}^{T-1}\{(\min (i / T, j / T)-i / T j / T) \\
& {\left.\left[P_{1, n}(i / T)-\hat{L}_{r, n, T} P_{2, r, n}(i / T)\right]\left[P_{1, n}(j / T)-\hat{L}_{r, n, T} P_{2, r, n}(j / T)\right]\left(x_{i+1: T}-x_{i: T}\right)\left(x_{j+1: T}-x_{j: T}\right)\right\}, }
\end{aligned}
$$

where:

$$
\hat{\lambda}_{2, r, n, T}=T^{-1} \sum_{t=1}^{T}\left[x_{t: T} P_{2, r, n}(t / T)\right]
$$

\section{Application to Hedge Funds}

In this section we derive and compare the rankings of hedge funds according to the unconditional Sharpe performance and a set of unconditional L-performance measures. The first section describes the set of pure hedge funds used in the application. The second section provides the summary statistics of fund return distributions to highlight the differences in fund behaviors, depending on their style. Finally, the third section compares the rankings obtained from the performance measures of interest.

\subsection{The Selected Funds}

The Hedge Fund Research, Inc. (HFR) database includes 3654 pure hedge funds and 1926 funds of funds, for which the return data are available for the period July 2004-June 2007. All returns are expressed in US dollars. They add up to a total value of $\$ 1400$ billion for both types of funds and $\$ 860$ billion for the pure hedge funds only.

At issuing, the hedge funds can be self-declared in one or several categories, called the styles, from a given list [see e.g. Das, Das (2004) for a description of styles in various databases]. These categories describe either the type of assets in the portfolio (as the styles "Currencies", "Distressed Securities"), or the type of portfolio management (for instance the styles "Global Macro", "Merger Arbitrage"), or both of these (as "Fixed Income Arbitrage", "Equity Long/Short"). A majority of pure hedge funds belong in 9 categories, that are "Equity Long/Short", "Fixed Income", "Global Macro", "Currency", "Futures", "Equity Long Short Equally Weighted", "Fixed Income Arbitrage", "Merger Arbitrage" and "Distressed Securities".

We select 36 funds from various categories and report the information on the management company, the self-declared strategy and the assets under management. This 
information is displayed in Table 1, where tickers are used to abbreviate the name of each fund in the sample.

[Insert Table 1: Funds by Strategy]

\subsection{Fund Return Distributions}

The unconditional return distribution of the hedge fund excess return is summarized by its conventional and L-moments of order 1 to 4 .

[Insert Table $2:$ L-Moments of order 1]

The first and last columns in Table 2 provide the mean and the median, respectively. Since the L-moments of order 1 are constructed from a basis of symmetric distortion polynomials, the distribution is symmetric if and only if all L-moments of order 1 are equal. For several funds such as CSS ED or DF MF, the distribution is almost symmetric, but in general the distribution of fund returns is skewed. We also note that a simple test of symmetry based on a comparison of the mean and the median can be misleading. For instance, the mean and median are rather close for funds IC EH or FX MA. We observe that the L-moments at order 1 in columns 2 to 6 are significantly smaller for fund IC $\mathrm{EH}$, whereas the L-moment for $n=2$ in column 3 is very high for fund FX MA, showing that the skewness concerns rather the central part of the distribution than the tails. This characteristic can reveal that some hedge fund managers avoid reporting losses to attract investors [Burgstahler, Dichev (1997), Asness, Krail, Liew (2001), Weinstein, Abdulali (2002)]. If some assets in a hedge fund portfolio are illiquid and if the portfolio manager uses some return smoothing techniques, then the return distribution can become skewed about zero. The manager can arbitrarily increase the number of small positive returns to have the number of small gains exceed the number of small losses [Bollen, Pool (2007), Figure 1]. Such data manipulation can be detected by measuring the departure of fund return distribution from a symmetric distribution in the neighbourhood of the median. The difference $\lambda_{1,2}-\lambda_{1,1}$ is a measure of this effect, and can be used as a test statistic in a test of manipulation. For example, an L-moment of order 1 with $n=2$ higher than the mean and the median is a warning that such a manipulation could have occurred for fund FX MA. The L-moment analysis is an alternative to the Bias Ratio, which compares the number of positive returns to the number of negative returns within one standard 
deviation of 0 [Abdulali (2006)]. It is more flexible, since it does not assume the existence of standard deviation and allows for different trimmings.

[Insert Table 3 : Power Moments and L-Moments of order 2]

The L-moments of order 2 for $n$ varying provide similar classifications of risks. For instance the fund RO ED, with the largest variance, has also the largest L-moments of order 2 for any value of trimming parameters. However, the funds next in the variance ranking, in a descending order such as funds DF MF, RC EH or WI MA, have a different ranking with respect to their L-moments of order 2. In particular, fund RC EH is more risky than WI MA in variance, but less risky in all L-moments of order 2 in columns 2-6.

[Insert Table 4 : Power Moments and L-Moments of order 3 and 4]

Table 4 provides the conventional skewness and excess kurtosis. It also displays the L-skewness and L-kurtosis defined by $\lambda_{3} / \lambda_{2}, \lambda_{4} / \lambda_{2}$ where: $\lambda_{3}=\frac{1}{3} E\left(\tilde{X}_{3: 3}-2 \tilde{X}_{2: 3}+\tilde{X}_{1: 3}\right)$, $\lambda_{4}=\frac{1}{4} E\left(\tilde{X}_{4: 4}-3 \tilde{X}_{3: 4}+3 \tilde{X}_{2: 4}-\tilde{X}_{1: 4}\right)$. The L-skewness (resp. L-kurtosis) measures the difference between the slopes of the quantile function for $u$ smaller and greater than the median, respectively, to detect asymmetries (resp. outside and within the quartile interval to detect the magnitude of the tail). The standard Gaussian L-kurtosis is equal to $\lambda_{4} / \lambda_{2}=\left[\frac{30}{\pi} \operatorname{arctg} \sqrt{2}-9\right] \frac{1}{\sqrt{\pi}}=0.0692$ [Hosking, Wallis (1997)]. As expected, the conventional and L-skewness of funds CSS ED and DF MF are close to zero (see the discussion of Table 2). More importantly, some of the funds which appear very risky in Table 3, with respect to their moments of order 2, can feature L-kurtosis smaller than the Gaussian L-kurtosis, which is equal to 0.0692. For example, funds WI MA or DF MF have large risks in the central part of the distribution, and rather small risks associated with the extremes.

The vertical and horizontal axes of Figure 4 measure the L-skewness and L-kurtosis, respectively. The set of admissible values of the L-skewness and L-kurtosis is the grey region above the lowest parabola [Hosking, Wallis (1997)]. The parabolic curves represent various 3-parameter families of distributions, and the points with coordinates $\mathrm{x}=$ sample L-skewness and $\mathrm{y}=\mathrm{L}$-kurtosis, respectively, represent the funds. Each curve represents a particular distribution family: glo (resp. gev, gpa, pe3) corresponds to the generalized logistic (resp. generalized extreme value, generalized Pareto, Pearson type III) distribution. 
Among the standard 3-parameter families, the generalized logistic distributions feature the heaviest tails. We observe that almost half of the funds have fatter tails than the generalized logistic.

[Insert Figure $4:$ L-skewness/kurtosis plot]

\subsection{Comparison of estimated performances}

In the next step, we compute the estimated Sharpe performance $\hat{S}_{T}$ and the estimated

L-performances $\hat{L}_{0,1, T}, \hat{L}_{1,2, T}, \hat{L}_{1,3, T}, \hat{L}_{1,4, T}, \hat{L}_{1,5, T}$. These statistics correspond to the conceptual samples of size $3,5,7,9,11$, respectively, and to ratios $r /(2 n+1)=\alpha$ equal to $0 \%, 20 \%, 14 \%, 11 \%, 9 \%$, respectively. The estimated performances and the associated rankings are provided in Table 5 .

[Insert Table 5 : Sharpe and L-Performances]

The values of different performance measures cannot be compared directly, as they do not have the same interpretation. In contrast, the rankings are comparable. We observe that the rankings based on the unconditional distributions are quite stable. For example, LES SS is always the worst performing fund, while FS EM is always the best performer. It is important to detect the hedge funds that have significantly changed their rankings. For example, the ranking of SP EH fluctuates between 3 and 15, which reveals the presence of extreme returns. This is largely due to the bias ratio observed in Table 2 with a jump in the L-moment of order 1 from $1.74 \%$ to $2.37 \%$, which is not strictly in line with the self-declared "Equity Hedge" strategy.

\section{Concluding Remarks}

The Sharpe ratio is commonly used in the hedge fund industry and provides a reliable performance measure for retail investors. However, the Sharpe ratio can be misleading for other types of investors such as banks, or regulators. Indeed, it is very sensitive to outliers, unable to correctly assess the sizes of tails that reflect extreme risks, or to detect manipulations of fund valuation. In the applied and theoretical literature, there exist alternative measures, such as the ratio mean/VaR(5\%) [see e.g. Dowd (1999), Gregoriou, Gueyle (2003)], the ratio $\operatorname{TVaR}(\alpha) / \operatorname{VaR}(\alpha)$, where TVaR denotes the so-called Tail-VaR 
[see e.g. Gourieroux, Liu (2007)]. The measures introduced in this paper have the same objective to improve upon the Sharpe ratio in these respects. The L-performances are based on the trimmed L-moment estimates to ensure the robustness and to account for thick tails. An additional feature of our measures is that the shrinkage parameters allow for fine-tunning of the L-performance measure that can produce a set of fund performances and rankings ${ }^{6}$. Such a set can be used to provide a comprehensive fund performance analysis and detect return smoothing and other manipulations by fund managers. They also provide alternative definitions of efficient portfolios and efficiency frontiers, formed by the L-performance maximizing portfolios, in the spirit of Roy [Roy (1952), see also Haly, McGee (2006)].

The literature on Sharpe performance measures would suggest to extend the methods proposed in this paper to the conditional and fitted performance measures, where the latter ones are suitable for hedge fund investments that completes another investment.

i) The conditional L-performance measure is computed from the conditional hedge fund excess return distribution. It can be obtained by estimating the conditional L-moments, i.e. by applying the conditional trimming proposed in Gourieroux, Jasiak (2008) for dynamic analysis of VaR. When the information is summarized by a single statistic, such as for instance the lagged market return, the L-performance can alternatively be computed from the concomitant ranks, that are the order statistics ranked according to the values of the lagged market return [Serfling, Xiao (2006)].

ii) The fitted performance is computed, for instance, when there is a portfolio of two assets, which are the fund and a market index (tracker). Then, it is necessary to disentangle the component of the hedge fund return which can be hedged by the market from its idiosyncratic component. The fitted L-performance is simply the L-performance applied to the residual plus intercept from the linear regression of hedge fund excess return on market excess return.

\footnotetext{
6" The numerical representations of funds require arrays because such funds represent sufficiently complicated objects that a single scalar most always proves inadequate at a summary level".
} 


\section{References}

[1] Abdulali, A. (2006): "The Bias Ratio: Measuring the Sharpe of Fraud", Protege Partners Quarterly Letter.

[2] Asness, C. Krail, R., and J., Liew (2001): "Do Hedge Funds Hedge?", Journal of Portfolio Mangement, 28, 6-19.

[3] Bayazit, M., and B., Onoz (2002) : "LL-Moments for Estimating Low Flow Quantiles", Hydrological Sciences, 47, 707-720.

[4] Bollen, N., and V., Pool (2007): "Do Hedge Fund Managers Misreport Returns? Evidence from the Pooled Distribution", Vanderbilt University, DP.

[5] Burgstahler, D., and I., Dichev (1997): "Earning Management to Avoid Earnings Decreases and Losses", Journal of Accounting and Economics, 24, 99-126.

[6] Darolles, S., and C., Gourieroux (2008): "Conditionally Fitted Sharpe Performance with an Application to Hedge Fund Rating", CREST, D.P.

[7] Das, N., and R., Das (2004): "Hedge Fund Classification Technique Using SelfOrganized Feature Map Neural Network", Working Paper.

[8] Dowd, K. (1999): "A Value-at-Risk Approach to Risk-Return Analysis", Journal of Portfolio Management, 25, 60-67.

[9] Elamir, E., and A., Seheult (2003): "Trimmed L-Moments", Computational Statistics and Data Analysis, 43, 299-314.

[10] Getmansky, M., Lo, A., and I., Makarov (2003): "An Econometric Model of Serial Correlation and Illiquidity in Hedge Fund Returns", MIT, Sloan School of Management, DP 4288-03.

[11] Gini, C. (1912): "Variabilita e Mutabilita, Contributo alla Studia della Distribuzioni e della Rezlaztione Statistiche", Stud. Econ. Univ. Cagl., 3, 3-159.

[12] Gourieroux, C., and J., Jasiak (2008): "Dynamic Quantile Model", Journal of Econometrics, forthcoming. 
[13] Gourieroux, C., and W., Liu (2007): "Efficient Portfolio Analysis Using Distorsion Risk Measures", HEC Montreal DP.

[14] Greenwood J., Landwehr, J., Matalas, N., and J., Wallis (1979): "Probability Weighted Moments: Definition and Relation to Parameters of Several Distributions Expressable in Inverse Forms", Water Resour. Res. 15, 1049-1054.

[15] Gregoriou, G., and J., Gueyle (2003): "Risk Adjusted Performance of Funds of Hedge Funds Using a Modified Sharpe Ratio", Journal of Wealth Management, Winter.

[16] Guttman, N., Hosking J., and J., Wallis (1993) : "Regional Precipitation Quantile Values for the Continental US Computed from L-Moments", J. Climate, 6, 2326-2340.

[17] Haley, R. and K., McGee (2006): "Tilting Safety First and the Sharpe Portfolio", Finance Research Letters, 3, 173-180.

[18] Hosking, J. (1990): "L-Moments: Analysis and Estimation of Distributions Using Linear Combinations of Order Statistics", JRSS B 52, 105-124.

[19] Hosking, J. (2006): "On the Characterization of Distributions by their L-Moments", Journal of Statistical Planning and Inference", 136, 193-198.

[20] Hosking, J. (2007): "Some Theory and Practical Uses of Trimmed L-Moments", Journal of Statistical Planning and Inference, 137, 3024-3039.

[21] Hosking, J., and J., Wallis (1987): "Parameter and Quantile Estimation for the Generalized Pareto Distribution", Technometrics, 29, 339-349.

[22] Jones, B., and R., Zitikis (2003): "Empirical Estimation of Risk Measures and Related Quantities", North American Actuarial Journal, 7, 44-54.

[23] Jones, B., and R., Zitikis (2005): "Testing for the Order of Risk Measures: An Application of L-Statistics in Actuarial Science", International Journal of Statistics, 63, 193-211.

[24] Karvanen, J. (2006): "Estimation of Quantile Mixtures via L-moments and Trimmed L-Moments", Computational Statistics and Data Analysis, 51, 947-959. 
[25] Koenker, R. (2005): "Quantile Regression", Cambridge University Press.

[26] Lee, S., and S., Maeng (2003): "Frequency Analysis of Extreme Rainfall Using LMoment", Irrig. and Drain, 52, 219-230.

[27] Lo, A. (2002): "The Statistics of Sharpe Ratio", Financial Analyst Journal, 58, 36-52.

[28] Moisello, U. (2007): "On the Use of Partial Probability Weighted Moments in the Analysis of Hydrological Extremes", Hydrol. Process, 21, 1265-1279.

[29] Roy, A. (1952): "Safety First and the Holding of Assets", Econometrica, 20, 431-439.

[30] Serfling, R. and P., Xiao (2007): "A Contribution to Multivariate L-moments : LComoment Matrices" , Journal of Multivariate Analysis, 98, 1765-1781.

[31] Sharpe, W. (1966): "Mutual Fund Performance", Journal of Business, 36, 119-138.

[32] Wang, Q. (1997):"LH-Moments for Statistical Analysis of Extreme Events", Water. Resour. Res., 33, 2841-2848.

[33] Wang, S. (2000a): "Premium Calculations by Transforming the Layer Premium Density", ASTIN Bulletin, 26, 71-92.

[34] Wang, S. (2000b): "A Class of Distortion Operators for Pricing Financial and Insurance Risks", Journal of Risk and Insurance, 67, 15-36.

[35] Weinstein, E., and A., Abdulali (2002): "Towards Hedge Fund Transparency: Quantifying Valuation Bias for Illiquid Assets", Risk, Spring.

[36] Whalen, T., Savage, G., and G., Jeong (2004): "An Evaluation of the Self-Determined Probability Weighted Moment Method for Estimating Extreme Wind Speeds", Journal of Wind Engineering and Industrial Aerodynamics, 92, 219-239. 


\section{Appendix 1}

\section{Asymptotic Properties of Estimated L-Performance}

\section{Asymptotic Properties of L-moments}

A trimmed L-moment is a linear form of the quantile fonction. It can be written as

$$
\lambda(Q, P)=\int_{0}^{1} Q(u) P(u) d u,
$$

where $P$ is a polynomial. It can consistently be estimated by the sample counterpart.

$$
\hat{\lambda}_{T}(Q, P)=\lambda\left(\hat{Q}_{T}, P\right)=\int_{0}^{1} \hat{Q}_{T}(u) P(u) d u,
$$

where $\hat{Q}_{T}(u)=\inf \left\{x: \frac{1}{T} \sum_{t=1}^{T} \mathbb{1}_{\left(X_{t} \leq x\right)} \geq u\right\}$ is the sample quantile function. This estimator is also equal to

$$
\hat{\lambda}_{T}(Q, P)=\frac{1}{T} \sum_{t=1}^{T} x_{t: T} P(t / T) .
$$

The asymptotic properties of the estimated L-moment are deduced from the asymptotic properties of the estimated quantile function.

The quantile estimator is related to the sample cdf by the Bahadur representation [see e.g. Koenker (2005), Section 4.3] :

$$
\sqrt{T}\left[\hat{Q}_{T}(u)-Q(u)\right]=-\frac{1}{f[Q(u)]} \sqrt{T}\left[\hat{F}_{T}(Q(u))-u\right]+o_{P}(1)
$$

where $\hat{F}_{T}(x)=\frac{1}{T} \sum_{t=1}^{T} \mathbb{1}_{x_{t} \leq x}$ is the sample cdf and $o_{P}(1)$ denotes a negligible term in probability. Moreover, it is known that $\sqrt{T}\left[\hat{F}_{T}(Q(u))-u\right]$ weakly converges to a Brownian bridge $B$ defined on $[0,1]$. Recall that a Brownian bridge is a Gaussian process on $[0,1]$, with zero-mean and covariance $\operatorname{Cov}\left[B\left(u_{1}\right), B\left(u_{2}\right)\right]=\min \left(u_{1}, u_{2}\right)-u_{1} u_{2}$. Thus, we deduce the (functional) limit theorem for the sample quantile :

$$
\sqrt{T}\left[\hat{Q}_{T}(u)-Q(u)\right] \Rightarrow-\frac{1}{f[Q(u)]} B(u),
$$

where $\Rightarrow$ denotes the weak convergence (convergence in distribution), and the asymptotic behaviour of a trimmed L-moment: 


$$
\begin{aligned}
& \sqrt{T}\left[\lambda\left(\hat{Q}_{T}, P\right)-\lambda(Q, P)\right]=\int_{0}^{1} \sqrt{T}\left[\hat{Q}_{T}(u)-Q(u)\right] P(u) d u \\
& \stackrel{d}{\rightarrow}-\int_{0}^{1} \frac{1}{f[Q(u)]} B(u) d u .
\end{aligned}
$$

In particular, the estimator $\lambda\left(\hat{Q}_{T}, P\right)$ is consistent, asymptotically Gaussian with zeromean and a variance given by :

$$
V_{a s}\left(\sqrt{T}\left[\lambda\left(\hat{Q}_{T}, P\right)-\lambda(Q, P)\right]\right)=\int_{0}^{1} \int_{0}^{1} \frac{\min \left(u_{1}, u_{2}\right)-u_{1} u_{2}}{f\left[Q\left(u_{1}\right)\right] f\left[Q\left(u_{2}\right)\right]} P\left(u_{1}\right) P\left(u_{2}\right) d u_{1} d u_{2} .
$$

\section{Application to estimated L-performance}

We have :

$$
\hat{L}_{r, n, T}=\hat{\lambda}_{1, n, T} / \hat{\lambda}_{2, r, n, T},
$$

where $\hat{\lambda}$ denotes the estimated L-moment. Since $\hat{\lambda}_{1, n, T}$ (resp. $\hat{\lambda}_{2, r, n, T}$ ) converges to $\lambda_{1, n}$ (resp. $\lambda_{2, r, n}$ ), and since $\lambda_{2, r, n}$ is strictly positive, we deduce that $\hat{L}_{r, n, T}$ tends to $L_{r, n}$.

Moreover, we have :

$$
\begin{aligned}
& \sqrt{T}\left(\hat{L}_{r, n, T}-L_{r, n}\right) \\
= & \sqrt{T}\left(\frac{\hat{\lambda}_{1, n, T}}{\hat{\lambda}_{2, r, n, T}}-\frac{\lambda_{1, n}}{\lambda_{2, r, n}}\right) \\
= & \frac{1}{\lambda_{2, r, n}} \sqrt{T}\left(\hat{\lambda}_{1, n, T}-\lambda_{1, n}\right)-\frac{\lambda_{1, n}}{\lambda_{2, r, n}^{2}} \sqrt{T}\left(\hat{\lambda}_{2, r, n, T}-\lambda_{2, r, n}\right)+o_{P}(1),
\end{aligned}
$$

(by the delta method)

$$
=\frac{1}{\lambda_{2, r, n}} \int_{0}^{1} \sqrt{T}\left[\hat{Q}_{T}(u)-Q(u)\right]\left[P_{1, n}(u)-L_{r, n} P_{2, n}(u)\right] d u .
$$

The expression of the asymptotic variance of the estimated L-performance directly follows from (A.1) applied to polynomial $P=\frac{1}{\lambda_{2, r, n}}\left(P_{1, n}-L_{r, n} P_{2, n}\right)$. 


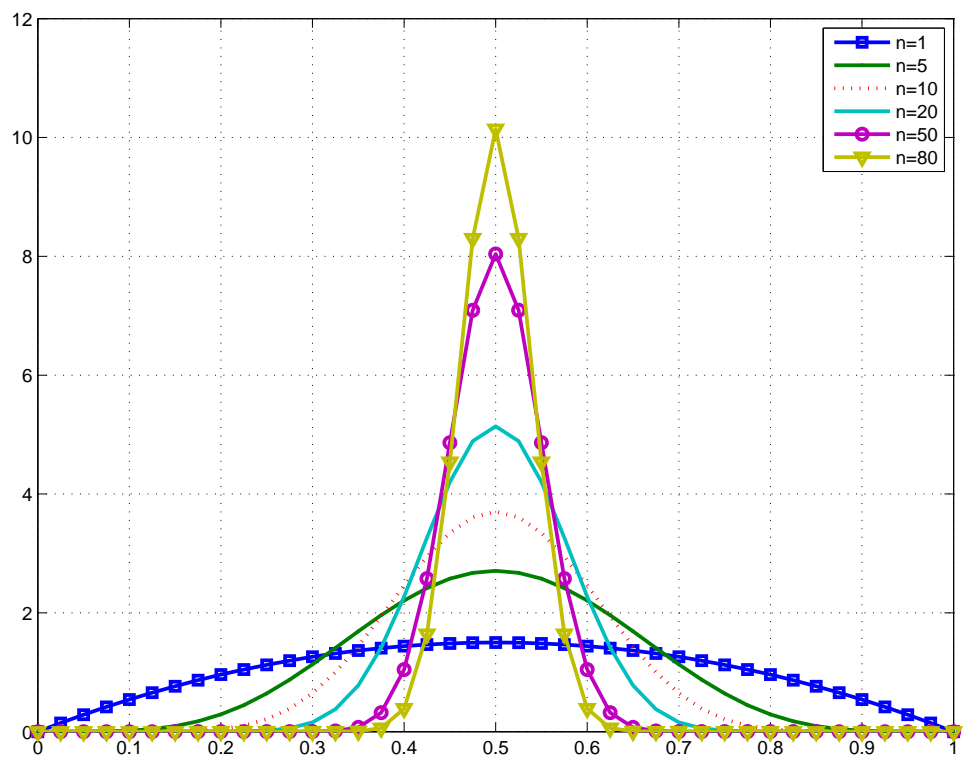

Figure 1: Weighting Polynomials $P_{1, n}$ 


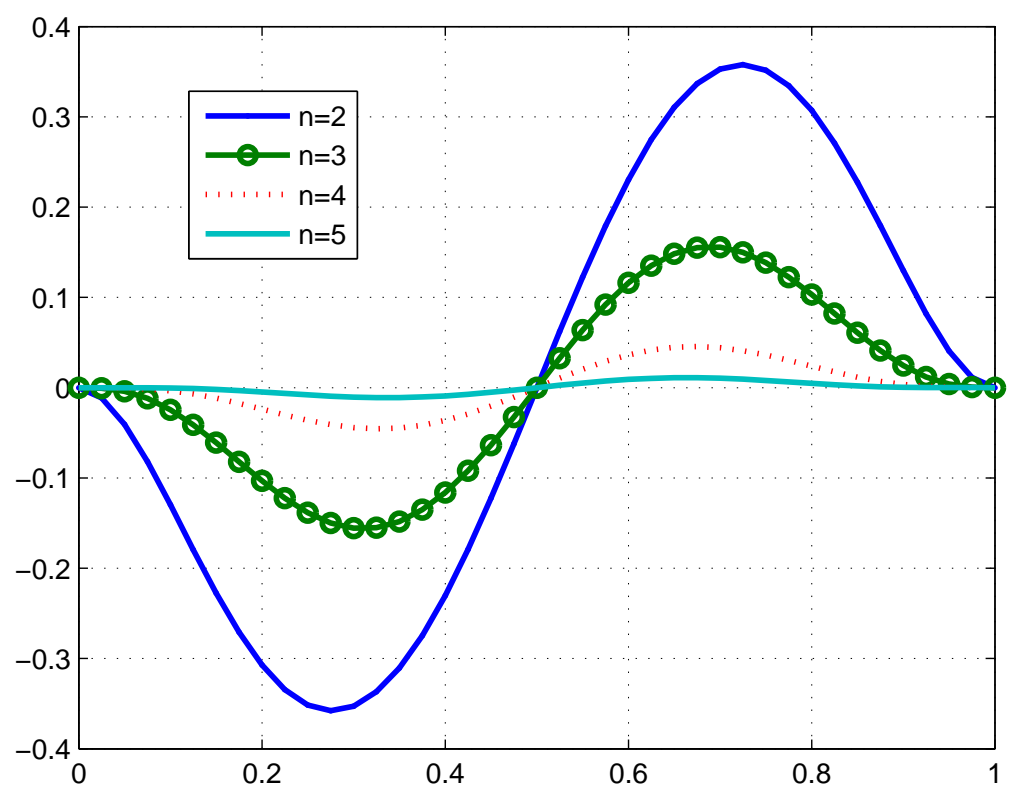

Figure 2: Polynomials $P_{2, r, n}$ for $r=1$ and $n$ varying 


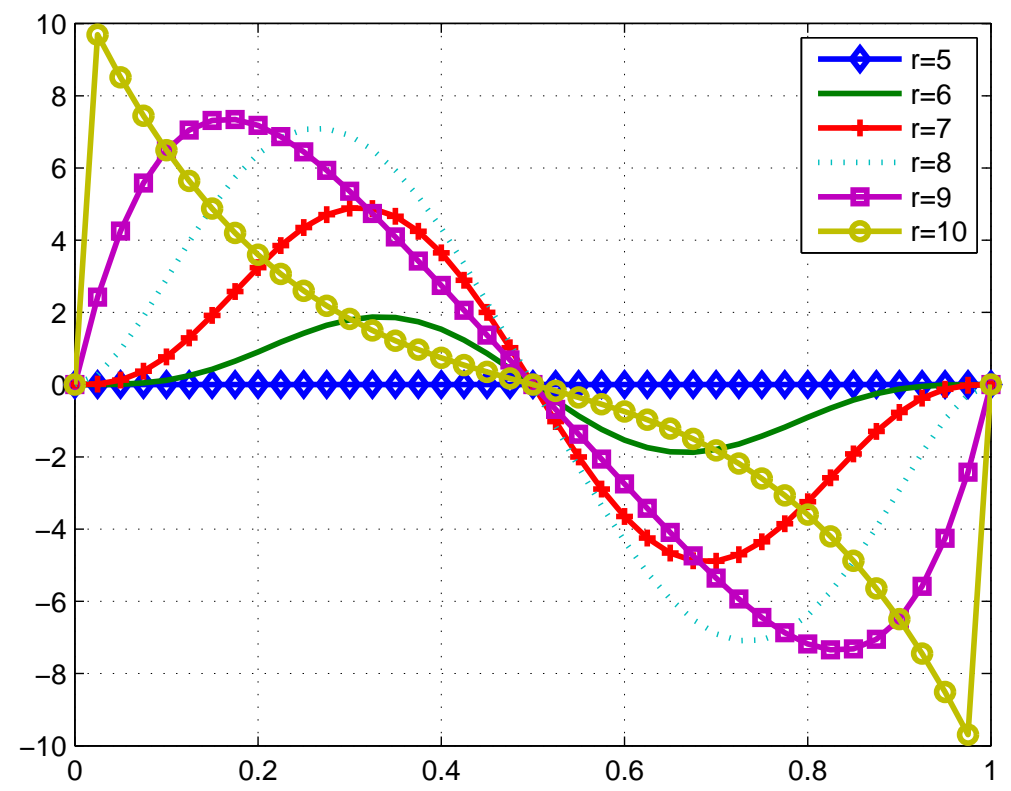

Figure 3: Polynomials $P_{2, r, n}$ for $n=5$ and $r$ varying 


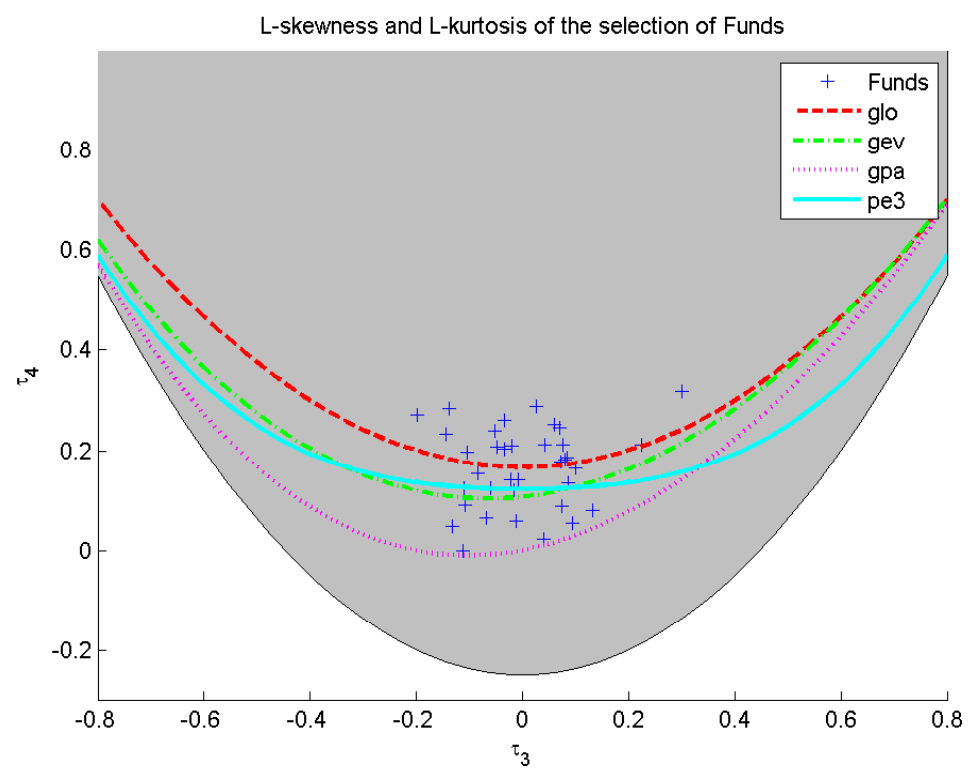

Figure 4: L-skewness/kurtosis plot 


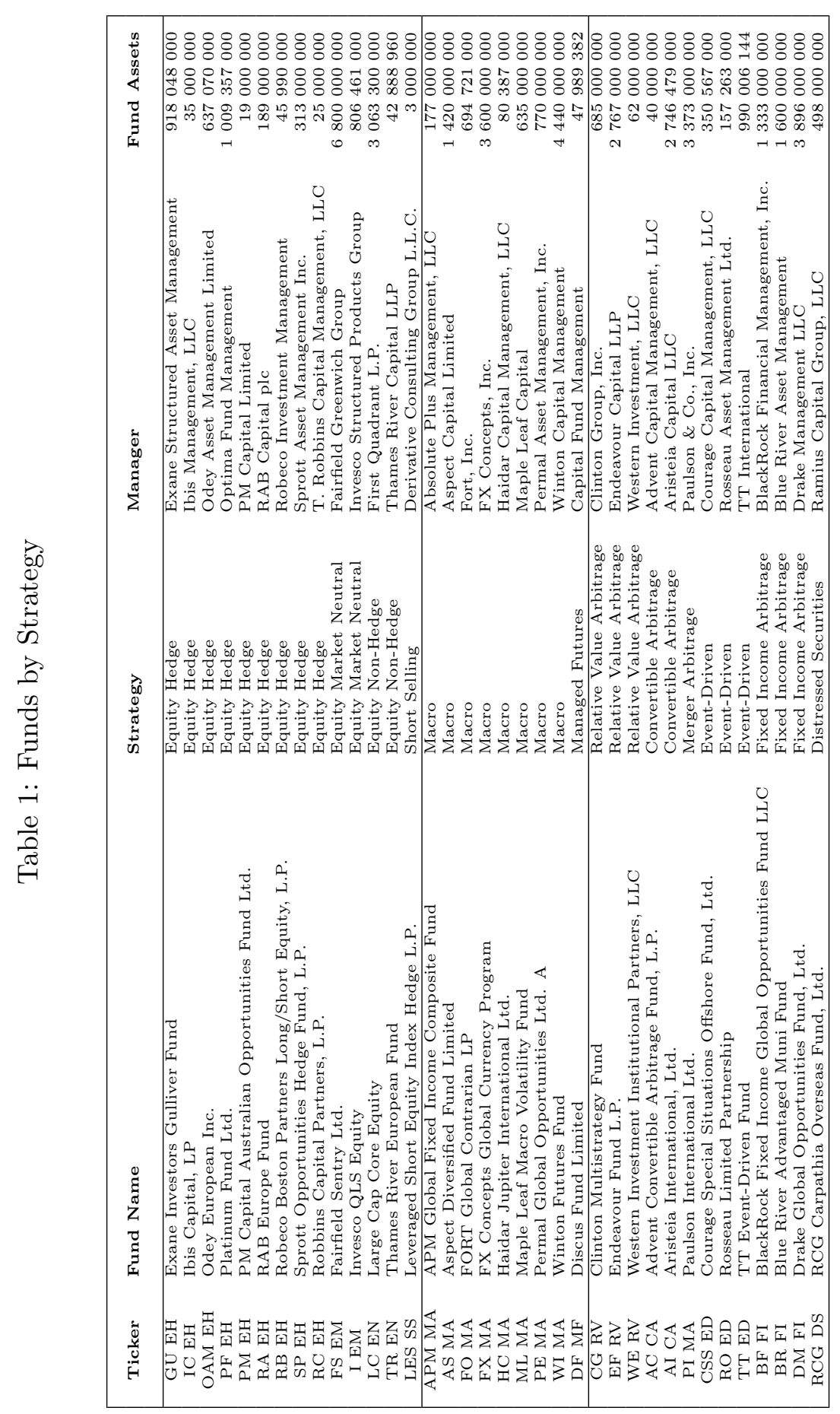


Table 2: L-Moments of order 1

\begin{tabular}{|c|c|c|c|c|c|c|c|}
\hline Ticker & $\lambda_{1, n}=0$ & $\lambda_{1, n=1}$ & $\lambda_{1, n=2}$ & $\lambda_{1, n=3}$ & $\lambda_{1, n=4}$ & $\lambda_{1, n=5}$ & $\lambda_{1, n}=\infty$ \\
\hline $\mathrm{AC} \mathrm{CA}$ & $0.056 \%$ & $0.027 \%$ & $0.078 \%$ & $0.071 \%$ & $0.081 \%$ & $0.087 \%$ & $0.202 \%$ \\
\hline AI CA & $0.371 \%$ & $0.292 \%$ & $0.414 \%$ & $0.305 \%$ & $0.308 \%$ & $0.310 \%$ & $0.325 \%$ \\
\hline APM MA & $0.546 \%$ & $0.419 \%$ & $0.538 \%$ & $0.359 \%$ & $0.333 \%$ & $0.311 \%$ & $0.276 \%$ \\
\hline AS MA & $0.814 \%$ & $0.906 \%$ & $1.349 \%$ & $0.986 \%$ & $0.978 \%$ & $0.962 \%$ & $0.601 \%$ \\
\hline BF FI & $-0.071 \%$ & $-0.065 \%$ & $-0.076 \%$ & $-0.050 \%$ & $-0.048 \%$ & $-0.046 \%$ & $-0.026 \%$ \\
\hline BR FI & $0.820 \%$ & $0.725 \%$ & $1.050 \%$ & $0.769 \%$ & $0.770 \%$ & $0.767 \%$ & $0.593 \%$ \\
\hline CG RV & $0.348 \%$ & $0.262 \%$ & $0.369 \%$ & $0.270 \%$ & $0.272 \%$ & $0.275 \%$ & $0.291 \%$ \\
\hline CSS ED & $0.499 \%$ & $0.436 \%$ & $0.615 \%$ & $0.448 \%$ & $0.450 \%$ & $0.452 \%$ & $0.443 \%$ \\
\hline DF MF & $2.344 \%$ & $2.138 \%$ & $2.994 \%$ & $2.174 \%$ & $2.182 \%$ & $2.187 \%$ & $2.256 \%$ \\
\hline DM FI & $1.072 \%$ & $0.770 \%$ & $1.010 \%$ & $0.712 \%$ & $0.704 \%$ & $0.701 \%$ & $0.781 \%$ \\
\hline EF RV & $0.042 \%$ & $-0.024 \%$ & $-0.023 \%$ & $-0.013 \%$ & $-0.012 \%$ & $-0.013 \%$ & $-0.164 \%$ \\
\hline FO MA & $0.909 \%$ & $0.838 \%$ & $1.145 \%$ & $0.814 \%$ & $0.805 \%$ & $0.800 \%$ & $0.865 \%$ \\
\hline FS EM & $0.303 \%$ & $0.260 \%$ & $0.357 \%$ & $0.259 \%$ & $0.262 \%$ & $0.264 \%$ & $0.304 \%$ \\
\hline FX MA & $0.859 \%$ & $1.016 \%$ & $1.533 \%$ & $1.128 \%$ & $1.127 \%$ & $1.116 \%$ & $0.883 \%$ \\
\hline GU EH & $0.197 \%$ & $0.215 \%$ & $0.319 \%$ & $0.235 \%$ & $0.236 \%$ & $0.236 \%$ & $0.228 \%$ \\
\hline HC MA & $0.806 \%$ & $0.873 \%$ & $1.351 \%$ & $1.033 \%$ & $1.069 \%$ & $1.095 \%$ & $1.252 \%$ \\
\hline I EM & $1.091 \%$ & $1.065 \%$ & $1.559 \%$ & $1.167 \%$ & $1.196 \%$ & $1.217 \%$ & $1.426 \%$ \\
\hline IC EH & $0.470 \%$ & $0.187 \%$ & $0.298 \%$ & $0.231 \%$ & $0.241 \%$ & $0.246 \%$ & $0.455 \%$ \\
\hline LC EN & $0.908 \%$ & $0.922 \%$ & $1.383 \%$ & $1.042 \%$ & $1.067 \%$ & $1.083 \%$ & $1.063 \%$ \\
\hline LES SS & $-1.076 \%$ & $-1.392 \%$ & $-2.045 \%$ & $-1.530 \%$ & $-1.564 \%$ & $-1.586 \%$ & $-1.584 \%$ \\
\hline ML MA & $0.460 \%$ & $0.373 \%$ & $0.525 \%$ & $0.370 \%$ & $0.359 \%$ & $0.348 \%$ & $0.309 \%$ \\
\hline OAM EH & $0.688 \%$ & $0.488 \%$ & $0.653 \%$ & $0.452 \%$ & $0.435 \%$ & $0.420 \%$ & $0.483 \%$ \\
\hline PE MA & $1.277 \%$ & $1.156 \%$ & $1.613 \%$ & $1.152 \%$ & $1.134 \%$ & $1.116 \%$ & $1.099 \%$ \\
\hline PF EH & $0.636 \%$ & $0.609 \%$ & $0.908 \%$ & $0.683 \%$ & $0.698 \%$ & $0.708 \%$ & $0.680 \%$ \\
\hline PI MA & $1.222 \%$ & $0.796 \%$ & $1.072 \%$ & $0.772 \%$ & $0.775 \%$ & $0.779 \%$ & $0.852 \%$ \\
\hline PM EH & $1.133 \%$ & $1.096 \%$ & $1.559 \%$ & $1.139 \%$ & $1.146 \%$ & $1.151 \%$ & $1.357 \%$ \\
\hline RA EH & $0.839 \%$ & $0.602 \%$ & $0.788 \%$ & $0.538 \%$ & $0.512 \%$ & $0.491 \%$ & $0.305 \%$ \\
\hline RCG DS & $0.907 \%$ & $0.694 \%$ & $0.947 \%$ & $0.681 \%$ & $0.681 \%$ & $0.682 \%$ & $0.956 \%$ \\
\hline RO ED & $2.695 \%$ & $2.477 \%$ & $3.641 \%$ & $2.735 \%$ & $2.808 \%$ & $2.860 \%$ & $3.539 \%$ \\
\hline RB EH & $0.758 \%$ & $0.736 \%$ & $1.046 \%$ & $0.760 \%$ & $0.761 \%$ & $0.761 \%$ & $0.799 \%$ \\
\hline SP EH & $2.132 \%$ & $1.744 \%$ & $2.373 \%$ & $1.718 \%$ & $1.732 \%$ & $1.749 \%$ & $2.100 \%$ \\
\hline $\mathrm{RC} \mathrm{EH}$ & $-0.540 \%$ & $-0.950 \%$ & $-1.392 \%$ & $-1.043 \%$ & $-1.071 \%$ & $-1.093 \%$ & $-1.164 \%$ \\
\hline TR EN & $1.180 \%$ & $1.199 \%$ & $1.787 \%$ & $1.337 \%$ & $1.365 \%$ & $1.385 \%$ & $1.563 \%$ \\
\hline TT ED & $1.031 \%$ & $0.986 \%$ & $1.421 \%$ & $1.046 \%$ & $1.058 \%$ & $1.066 \%$ & $1.399 \%$ \\
\hline WE RV & $0.611 \%$ & $0.461 \%$ & $0.605 \%$ & $0.428 \%$ & $0.425 \%$ & $0.426 \%$ & $0.510 \%$ \\
\hline WI MA & $1.197 \%$ & $1.241 \%$ & $1.901 \%$ & $1.462 \%$ & $1.528 \%$ & $1.581 \%$ & $2.237 \%$ \\
\hline
\end{tabular}


Table 3: Power Moments and L-Moments of order 2

\begin{tabular}{|c|c|c|c|c|c|c|}
\hline \multirow[b]{2}{*}{ Ticker } & \multirow{2}{*}{$\begin{array}{c}\text { Power Moments } \\
\sigma\end{array}$} & \multicolumn{5}{|c|}{ L-Moments } \\
\hline & & $\lambda_{2, r}=0, n=1$ & $\lambda_{2, r}=1, n=2$ & $\lambda_{2, r}=1, n=3$ & $\lambda_{2, r}=1, n=4$ & $\lambda_{2, r=1, n=5}$ \\
\hline AC CA & $1.14 \%$ & $0.151 \%$ & $0.138 \%$ & $0.047 \%$ & $0.012 \%$ & $0.003 \%$ \\
\hline $\mathrm{AI} C A$ & $0.95 \%$ & $0.140 \%$ & $0.131 \%$ & $0.046 \%$ & $0.011 \%$ & $0.003 \%$ \\
\hline APM MA & $1.90 \%$ & $0.313 \%$ & $0.297 \%$ & $0.103 \%$ & $0.026 \%$ & $0.006 \%$ \\
\hline AS MA & $3.63 \%$ & $0.589 \%$ & $0.556 \%$ & $0.194 \%$ & $0.049 \%$ & $0.011 \%$ \\
\hline BF FI & $0.30 \%$ & $0.041 \%$ & $0.036 \%$ & $0.012 \%$ & $0.003 \%$ & $0.001 \%$ \\
\hline BR FI & $2.80 \%$ & $0.348 \%$ & $0.288 \%$ & $0.092 \%$ & $0.022 \%$ & $0.005 \%$ \\
\hline CG RV & $1.22 \%$ & $0.146 \%$ & $0.123 \%$ & $0.040 \%$ & $0.010 \%$ & $0.002 \%$ \\
\hline CSS ED & $1.42 \%$ & $0.209 \%$ & $0.191 \%$ & $0.066 \%$ & $0.017 \%$ & $0.004 \%$ \\
\hline DF MF & $4.88 \%$ & $0.785 \%$ & $0.728 \%$ & $0.249 \%$ & $0.062 \%$ & 0. $013 \%$ \\
\hline DM FI & $3.38 \%$ & $0.458 \%$ & $0.395 \%$ & $0.130 \%$ & $0.032 \%$ & $0.007 \%$ \\
\hline EF RV & $1.24 \%$ & $0.167 \%$ & $0.147 \%$ & $0.049 \%$ & $0.012 \%$ & $0.003 \%$ \\
\hline FO MA & $1.87 \%$ & $0.249 \%$ & $0.215 \%$ & $0.071 \%$ & $0.017 \%$ & $0.004 \%$ \\
\hline FS EM & $0.41 \%$ & $0.056 \%$ & $0.051 \%$ & $0.017 \%$ & $0.004 \%$ & $0.001 \%$ \\
\hline FX MA & $3.09 \%$ & $0.377 \%$ & $0.306 \%$ & $0.096 \%$ & $0.023 \%$ & $0.005 \%$ \\
\hline GU EH & $0.71 \%$ & $0.084 \%$ & $0.068 \%$ & $0.022 \%$ & $0.005 \%$ & $0.001 \%$ \\
\hline HC MA & $2.26 \%$ & $0.295 \%$ & $0.255 \%$ & $0.084 \%$ & $0.020 \%$ & $0.004 \%$ \\
\hline I EM & $2.24 \%$ & $0.357 \%$ & $0.328 \%$ & $0.111 \%$ & $0.027 \%$ & $0.006 \%$ \\
\hline IC EH & $3.36 \%$ & $0.409 \%$ & $0.357 \%$ & $0.118 \%$ & $0.029 \%$ & $0.006 \%$ \\
\hline LC EN & $2.35 \%$ & $0.365 \%$ & $0.328 \%$ & $0.108 \%$ & $0.026 \%$ & $0.005 \%$ \\
\hline LES SS & $3.23 \%$ & $0.510 \%$ & $0.471 \%$ & $0.160 \%$ & $0.039 \%$ & $0.008 \%$ \\
\hline ML MA & $1.67 \%$ & $0.191 \%$ & $0.160 \%$ & $0.052 \%$ & $0.012 \%$ & $0.003 \%$ \\
\hline OAM EH & $2.66 \%$ & $0.366 \%$ & $0.327 \%$ & $0.111 \%$ & $0.027 \%$ & $0.006 \%$ \\
\hline PE MA & $3.03 \%$ & $0.447 \%$ & $0.412 \%$ & $0.142 \%$ & $0.036 \%$ & $0.008 \%$ \\
\hline PF EH & $2.04 \%$ & $0.297 \%$ & $0.260 \%$ & $0.086 \%$ & $0.021 \%$ & 0. $004 \%$ \\
\hline PI MA & $2.53 \%$ & $0.234 \%$ & $0.205 \%$ & $0.069 \%$ & $0.017 \%$ & 0. $004 \%$ \\
\hline PM EH & $1.69 \%$ & $0.247 \%$ & $0.224 \%$ & $0.077 \%$ & $0.019 \%$ & 0. $004 \%$ \\
\hline RA EH & $2.65 \%$ & $0.330 \%$ & $0.294 \%$ & $0.099 \%$ & $0.024 \%$ & $0.005 \%$ \\
\hline RCG DS & $2.11 \%$ & $0.285 \%$ & $0.258 \%$ & $0.088 \%$ & $0.022 \%$ & $0.005 \%$ \\
\hline RO ED & $5.93 \%$ & $0.883 \%$ & $0.812 \%$ & $0.279 \%$ & $0.069 \%$ & 0. $015 \%$ \\
\hline RB EH & $1.67 \%$ & $0.215 \%$ & $0.182 \%$ & $0.060 \%$ & $0.015 \%$ & $0.003 \%$ \\
\hline SP EH & $3.31 \%$ & $0.496 \%$ & $0.471 \%$ & $0.166 \%$ & $0.042 \%$ & $0.009 \%$ \\
\hline RC EH & $4.88 \%$ & $0.654 \%$ & $0.582 \%$ & $0.195 \%$ & $0.048 \%$ & 0. $010 \%$ \\
\hline TR EN & $2.74 \%$ & $0.376 \%$ & $0.333 \%$ & $0.111 \%$ & $0.027 \%$ & $0.006 \%$ \\
\hline TT ED & $2.20 \%$ & $0.292 \%$ & $0.251 \%$ & $0.083 \%$ & $0.020 \%$ & $0.004 \%$ \\
\hline WE RV & $1.05 \%$ & $0.132 \%$ & $0.113 \%$ & $0.036 \%$ & $0.009 \%$ & $0.002 \%$ \\
\hline WI MA & $4.21 \%$ & $0.718 \%$ & $0.691 \%$ & $0.244 \%$ & $0.062 \%$ & $0.014 \%$ \\
\hline
\end{tabular}


Table 4: Power Moments and L-Moments of order 3 and 4

\begin{tabular}{|c|cc|cc|}
\hline & \multicolumn{2}{|c|}{ Power Moments } & \multicolumn{2}{|c|}{ L Moments } \\
Ticker & Skewness & Kurtosis & L-skewness & L-kurtosis \\
\hline AC CA & 0.12 & 1.49 & -0.03 & 0.20 \\
AI CA & 0.61 & 0.80 & 0.07 & 0.09 \\
APM MA & 0.14 & -1.12 & 0.04 & 0.02 \\
AS MA & -0.55 & -0.50 & -0.13 & 0.05 \\
BF FI & -0.49 & 1.43 & -0.11 & 0.12 \\
BR FI & -0.14 & 0.81 & -0.03 & 0.26 \\
CG RV & 0.34 & 1.45 & 0.06 & 0.25 \\
CSS ED & -0.04 & -0.14 & -0.01 & 0.14 \\
DF MF & -0.04 & -0.89 & -0.01 & 0.06 \\
DM FI & 0.38 & 0.17 & 0.08 & 0.19 \\
EF RV & 0.11 & 0.41 & 0.04 & 0.21 \\
FO MA & -0.40 & 0.90 & -0.02 & 0.21 \\
FS EM & 0.62 & 0.47 & 0.09 & 0.13 \\
FX MA & -1.23 & 2.03 & -0.20 & 0.27 \\
GU EH & -1.16 & 2.68 & -0.14 & 0.28 \\
HC MA & -0.62 & 0.90 & -0.14 & 0.23 \\
I EM & -0.24 & -0.81 & -0.07 & 0.06 \\
IC EH & 0.92 & 2.82 & 0.07 & 0.25 \\
LC EN & -0.39 & -0.64 & -0.11 & 0.09 \\
LES SS & 0.36 & -0.87 & 0.09 & 0.05 \\
ML MA & 0.30 & 2.83 & 0.03 & 0.29 \\
OAM EH & 0.33 & 0.58 & 0.07 & 0.18 \\
PE MA & -0.08 & -0.06 & -0.02 & 0.14 \\
PF EH & -0.26 & -0.24 & -0.08 & 0.15 \\
PI MA & 2.78 & 11.03 & 0.30 & 0.32 \\
PM EH & -0.18 & -0.03 & -0.06 & 0.13 \\
RA EH & 0.62 & 2.65 & 0.08 & 0.21 \\
RCG DS & 0.72 & 1.14 & 0.10 & 0.16 \\
RO ED & 0.06 & -0.25 & -0.02 & 0.12 \\
RB EH & -0.51 & 1.07 & -0.05 & 0.24 \\
SP EH & 0.66 & 0.07 & 0.13 & 0.08 \\
RC EH & 0.24 & 0.98 & 0.08 & 0.18 \\
TR EN & -0.38 & 0.63 & -0.11 & 0.20 \\
TT ED & -0.28 & 0.53 & -0.05 & 0.21 \\
WE RV & 1.02 & 1.06 & 0.23 & 0.21 \\
WI MA & -0.36 & -1.16 & -0.11 & -0.00 \\
\hline & & & & \\
\hline
\end{tabular}


Table 5: Sharpe and L-Performances

\begin{tabular}{|c|c|c|c|c|c|c|c|c|c|c|c|c|c|c|}
\hline \multirow[b]{2}{*}{ Ticker } & \multicolumn{4}{|c|}{ Sharpe Performance } & \multicolumn{10}{|c|}{ L-Performances } \\
\hline & SR & Rank & Skew. & Kurt. & $L_{0,1}$ & Rank & $L_{1,2}$ & Rank & $L_{1,3}$ & Rank & $L_{1,4}$ & Rank & $L_{1,5}$ & Rank \\
\hline $\mathrm{AC} \mathrm{CA}$ & 0.05 & 32 & 0.12 & 1.49 & 0.18 & 32 & 0.57 & 32 & 1.51 & 32 & 6.80 & 32 & 33.68 & 32 \\
\hline AI CA & 0.39 & 15 & 0.61 & 0.80 & 2.09 & 19 & 3.15 & 23 & 6.66 & 24 & 26.79 & 24 & 123.60 & 24 \\
\hline APM MA & 0.29 & 23 & 0.14 & -1.12 & 1.34 & 29 & 1.81 & 30 & 3.48 & 30 & 12.95 & 30 & 55.81 & 30 \\
\hline AS MA & 0.22 & 30 & -0.55 & -0.50 & 1.54 & 28 & 2.43 & 28 & 5.08 & 28 & 19.97 & 28 & 89.86 & 28 \\
\hline BF FI & -0.24 & 35 & -0.49 & 1.43 & -1.61 & 35 & -2.10 & 34 & -4.12 & 34 & -15.94 & 34 & -71.74 & 34 \\
\hline BR FI & 0.29 & 22 & -0.14 & 0.81 & 2.08 & 21 & 3.65 & 19 & 8.33 & 17 & 34.67 & 17 & 162.40 & 17 \\
\hline CG RV & 0.28 & 25 & 0.34 & 1.45 & 1.79 & 25 & 3.00 & 24 & 6.74 & 23 & 28.02 & 22 & 132.30 & 21 \\
\hline CSS ED & 0.35 & 18 & -0.04 & -0.14 & 2.09 & 20 & 3.22 & 22 & 6.78 & 22 & 27.07 & 23 & 124.04 & 23 \\
\hline DF MF & 0.48 & 8 & -0.04 & -0.89 & 2.72 & 13 & 4.11 & 16 & 8.72 & 16 & 35.16 & 16 & 162.72 & 16 \\
\hline DM FI & 0.32 & 19 & 0.38 & 0.17 & 1.68 & 27 & 2.56 & 27 & 5.48 & 26 & 22.21 & 26 & 103.29 & 26 \\
\hline EF RV & 0.03 & 33 & 0.11 & 0.41 & -0.14 & 33 & -0.16 & 33 & -0.27 & 33 & -1.02 & 33 & -4.85 & 33 \\
\hline FO MA & 0.49 & 5 & -0.40 & 0.90 & 3.36 & 8 & 5.34 & 7 & 11.53 & 9 & 46.88 & 9 & 218.13 & 9 \\
\hline FS EM & 0.74 & 1 & 0.62 & 0.47 & 4.63 & 1 & 7.06 & 1 & 15.14 & 1 & 61.77 & 1 & 289.08 & 1 \\
\hline FX MA & 0.28 & 26 & -1.23 & 2.03 & 2.70 & 14 & 5.00 & 11 & 11.71 & 8 & 49.29 & 7 & 232.30 & 7 \\
\hline GU EH & 0.28 & 27 & -1.16 & 2.68 & 2.56 & 16 & 4.67 & 13 & 10.81 & 11 & 45.53 & 11 & 215.93 & 10 \\
\hline HC MA & 0.36 & 17 & -0.62 & 0.90 & 2.96 & 11 & 5.30 & 8 & 12.33 & 5 & 52.69 & 3 & 253.63 & 3 \\
\hline I EM & 0.49 & 6 & -0.24 & -0.81 & 2.98 & 10 & 4.75 & 12 & 10.50 & 12 & 43.81 & 12 & 208.01 & 12 \\
\hline IC EH & 0.14 & 31 & 0.92 & 2.82 & 0.46 & 31 & 0.84 & 31 & 1.95 & 31 & 8.28 & 31 & 39.50 & 31 \\
\hline LC EN & 0.39 & 16 & -0.39 & -0.64 & 2.53 & 17 & 4.22 & 15 & 9.65 & 15 & 41.04 & 14 & 197.20 & 13 \\
\hline LES SS & -0.33 & 36 & 0.36 & -0.87 & -2.73 & 36 & -4.34 & 36 & -9.56 & 36 & -39.64 & 36 & -187.19 & 36 \\
\hline ML MA & 0.28 & 28 & 0.30 & 2.83 & 1.96 & 23 & 3.27 & 21 & 7.15 & 21 & 28.82 & 21 & 131.98 & 22 \\
\hline OAM EH & 0.26 & 29 & 0.33 & 0.58 & 1.34 & 30 & 2.00 & 29 & 4.09 & 29 & 15.90 & 29 & 71.06 & 29 \\
\hline PE MA & 0.42 & 14 & -0.08 & -0.06 & 2.58 & 15 & 3.91 & 17 & 8.09 & 18 & 31.71 & 19 & 142.90 & 19 \\
\hline PF EH & 0.31 & 21 & -0.26 & -0.24 & 2.05 & 22 & 3.49 & 20 & 7.94 & 19 & 33.31 & 18 & 157.89 & 18 \\
\hline PI MA & 0.48 & 7 & 2.78 & 11.03 & 3.40 & 6 & 5.22 & 9 & 11.24 & 10 & 45.74 & 10 & 212.83 & 11 \\
\hline PM EH & 0.67 & 2 & -0.18 & -0.03 & 4.43 & 2 & 6.95 & 2 & 14.80 & 2 & 59.41 & 2 & 273.04 & 2 \\
\hline RA EH & 0.32 & 20 & 0.62 & 2.65 & 1.82 & 24 & 2.68 & 26 & 5.44 & 27 & 21.07 & 27 & 94.08 & 27 \\
\hline RCG DS & 0.43 & 13 & 0.72 & 1.14 & 2.44 & 18 & 3.67 & 18 & 7.70 & 20 & 30.81 & 20 & 141.79 & 20 \\
\hline RO ED & 0.45 & 10 & 0.06 & -0.25 & 2.81 & 12 & 4.48 & 14 & 9.82 & 14 & 40.46 & 15 & 189.94 & 14 \\
\hline RB EH & 0.45 & 11 & -0.51 & 1.07 & 3.42 & 5 & 5.73 & 3 & 12.68 & 3 & 51.92 & 4 & 241.81 & 5 \\
\hline SP EH & 0.64 & 3 & 0.66 & 0.07 & 3.52 & 3 & 5.04 & 10 & 10.35 & 13 & 41.12 & 13 & 188.94 & 15 \\
\hline $\mathrm{RC} \mathrm{EH}$ & -0.11 & 34 & 0.24 & 0.98 & -1.45 & 34 & -2.39 & 35 & -5.35 & 35 & -22.49 & 35 & -107.53 & 35 \\
\hline TR EN & 0.43 & 12 & -0.38 & 0.63 & 3.19 & 9 & 5.37 & 5 & 12.07 & 6 & 50.39 & 6 & 238.68 & 6 \\
\hline TT ED & 0.47 & 9 & -0.28 & 0.53 & 3.37 & 7 & 5.66 & 4 & 12.58 & 4 & 51.78 & 5 & 242.00 & 4 \\
\hline WE RV & 0.58 & 4 & 1.02 & 1.06 & 3.49 & 4 & 5.36 & 6 & 11.74 & 7 & 48.79 & 8 & 231.91 & 8 \\
\hline WI MA & 0.28 & 24 & -0.36 & -1.16 & 1.73 & 26 & 2.75 & 25 & 6.00 & 25 & 24.80 & 25 & 117.06 & 25 \\
\hline
\end{tabular}

